\title{
Prospecção Científica e Tecnológica de Malpighia emarginata DC. (Malpighiaceae): espécie economicamente importante
}

\section{Scientific and Technological Prospection of Malpighia emarginata DC. (Malpighiaceae): economically important specie}

\author{
Thaynara de Carvalho Vieira ${ }^{1}$ \\ Maria Gracelia Paiva Nascimento ${ }^{2}$ \\ Cleiton Barroso Bittencourt ${ }^{3}$ \\ Ivanilza Moreira de Andrade ${ }^{1}$ \\ ${ }^{1}$ Universidade Federal do Delta do Parnaíba, Parnaîba, PI, Brasil \\ ${ }^{2}$ Universidade Federal do Piauí, Teresina, PI, Brasil \\ ${ }^{3}$ Universidade Federal de Lavras, Lavras, MG, Brasil
}

\begin{abstract}
Resumo
Malpighia emarginata DC. é uma espécie frutífera com ampla distribuição no Brasil e potencial econômico no campo alimentício, principalmente como fonte de Vitamina C. Neste trabalho, realizou-se uma prospecção científica $e$ tecnológica em bases de dados de artigos e patentes, nacional e internacional, sobre Malpighia emarginata. Na Web of Science e no SCOPUS foram indexados 169 e 179 artigos, respectivamente, enquanto, para patentes, foram obtidos 11 (EPO) e 20 (WIPO) registros. Com relação a países, a China $(\mathrm{EPO}=5$ e WIPO=6) e a Coreia do Sul $(\mathrm{EPO}=3$ $e \mathrm{WIPO}=4)$ são os maiores detentores de patentes envolvendo o desenvolvimento de pesquisas sobre Malpighia emarginata. As pesquisas tecnológicas e científicas sugerem que o gênero Malpighia tornou-se bastante conhecido principalmente devido às suas propriedades nutricionais, e, embora o Brasil possua um número significativo de publicações, é necessário que haja maior produção de tecnologias, devido ao grande avanço técnico científico para a espécie.
\end{abstract}

Palavras-chave: Malpighia emarginata. Produção Científica. Prospecção Tecnológica.

\begin{abstract}
Malpighia emarginata DC. is a fruit species that is widely distributed in Brazil and economically important as a food source, particularly as a source of vitamin C. A literature search was carried out for scientific and technological articles and patents on Malpighia emarginata. In Web of Science and SCOPUS 169 and 179 articles were indexed respectively while for patents 11 (EPO) and 20 (WIPO) records were found. In regard to national contribution, China (EPO=5, $\mathrm{WIPO}=6)$ and South Korea $(\mathrm{EPO}=3, \mathrm{WIPO}=4)$ are the major holders of patents based on research development in Malpighia emarginata. Technological research and development suggest that the genus Malpighia is now well known because of its nutritional properties and although Brazil has produced a significant number of publications, greater technological production is needed, given the major technical and scientific advances that have been made in regard to this species.
\end{abstract}

Keywords: Malpighia emarginata. Scientific Production. Technological Prospecting.

Área Tecnológica: Prospecção Científica e Tecnológica. 


\section{Introdução}

Malpighia emarginata DC. (Malpighiaceae), popularmente conhecida como acerola, é uma espécie originária da América Central, Noroeste da América do Sul e Antilhas (SHINOHARA et al., 2015). Apresenta porte arbustivo e, quando presente em regiões de clima tropical, frutifica praticamente o ano todo (SILVA et al., 2016). Foi introduzida no Brasil por volta dos anos de 1950 e distribui-se em todo território nacional (MENEZES et al., 2009; DE ALMEIDA et al., 2017). As condições climáticas favoráveis ao seu cultivo colocaram o Brasil como o maior produtor e exportador dos insumos advindos da aceroleira (FURLANETO; NASSER , 2015).

Espécies vegetais têm contribuído desde os primórdios como fonte de substâncias úteis ao tratamento de doenças que acometem o ser humano (BELWAL et al., 2018). Logo, a indústria farmacêutica, motivada pela descoberta e uso de quimioterápicos eficazes, começou a aplicar grandes investimentos em pesquisa de bioprospecção com o objetivo de rastrear novos fármacos com custo racional (DELVA; GOODRICH-SCHNEIDER, 2013). Assim, já é relatado na literatura a aplicabilidade de M. emarginata, a qual foi reportada a capacidade antioxidante, além de metabólitos secundários presentes em seus frutos, que possuem grande potencial antimicrobiano (REZENDE; NOGUEIRA; NARAIN, 2017).

Mesmo com a ampla presença de compostos que podem ser empregados em diversas áreas, por exemplo, na área médica (SILVA et al., 2015), o valor comercial da espécie na área alimentícia impulsiona pesquisas que visam ao aumento da produção de vitamina $C$, à utilização de caracteres fisiológicos, bioquímicos, aspectos nutricionais, além do emprego de marcadores moleculares para o seu melhoramento genético (OLIVEIRA et al., 2009; DA CRUZ et al, 2011; FREITAS et al., 2014). Com isso, a versatilidade de uso de Malpighia emarginata em diversas áreas colocam o grupo em destaque como fonte de tecnologias ainda pouco conhecidas (NOGUEIRA et al., 2019).

Nesse contexto, a busca por inovações tecnológicas que impulsionam o desenvolvimento tende a auxiliar o aumento da produtividade e a geração de ganhos econômicos para empresas nos mais diversos setores industriais do Brasil e do mundo (ACIOLI; ABUD; JÚNIOR, 2015). Dessa forma, os estudos prospectivos são de grande importância, pois as bases de dados tecnológicas e científicas nacionais e internacionais vêm crescendo significativamente nas últimas décadas e são fontes de informações valiosas sobre diversas áreas e de rastreio de aplicabilidade de novas tecnologias (MARQUES et al., 2013; JUNIOR et al., 2014; SPÓSITO; VIRGENS; PUNGARTNIK, 2016), ampliando, dessa forma, a capacidade de antecipação (QUINTELLA et al., 2011).

Diante do exposto, objetivou-se realizar uma prospecção tecnológica sobre a espécie Malphigia emarginata, analisando o número de registros de depósitos de patentes por países, ano de depósito e suas classificações internacionais (CIP), utilizando, para tanto, bancos de dados de inovação e tecnologia nacional e internacional com o intuito de descrever o perfil da produção científica nas áreas de aplicação com base em artigos científicos. 


\section{Metodologia}

A pesquisa foi realizada em dezembro de 2019 e, para a prospecção científica, foram utilizados oito descritores ("Malpighiaceae", "Malpighia emarginata", "plant AND food", "plant AND genetic", "plant AND production", "Malpighia emarginata AND food", "Malpighia emarginata AND genética" e "Malpighia emarginata AND produção") com aspas para maior precisão.

Este trabalho é uma pesquisa documental exploratória de abordagem quantitativa. A prospecção foi realizada nas bases de dados científicos da Web of Science (WoS) e SCOPUS e nos bancos de patentes do Instituto Nacional da Propriedade Industrial (INPI) do Brasil no Banco Europeu de Patentes (EPO) e no Banco da Organização Mundial da Propriedade Intelectual (OMPI).

O descritor que apresentou o maior número de citações na prospecção científica foi utilizado na prospecção tecnológica na base de dados mundial: World Intellectual Property Organization (WIPO), base europeia European Patent Office (EPO) e na base brasileira do Instituto Nacional da Propriedade Industrial (INPI).

Os termos em português foram utilizados para a busca de documentos em base nacional, enquanto os termos em inglês foram utilizados para as bases internacionais, sendo considerados válidos os documentos que apresentassem esses termos no título e/ou no resumo. Optou-se por utilizar nesta pesquisa, para a seleção os dados bibliográficos, a base Web of Science (WoS) para elaboração dos gráficos. Essa escolha se deu devido à grande disponibilidade de trabalhos científicos e por ser a base que dá origem ao fator de impacto dos periódicos (JCR - Journal Citation Report). Os dados foram tabulados de forma individual, conforme as categorias de análises (país, área, espécies e ano) e organizados em tabelas e gráficos no Microsoft Excel 2016, visando à caracterização, distribuição e avanço das publicações e os pedidos de patente existentes até o presente momento. As bases que obtiveram os resultados mais significativos tiveram seus resultados separados por ano de publicação, países, áreas de conhecimento e palavras-chave para melhor caracterização, distribuição e avanço das publicações existentes até julho de 2019.

\section{Resultados e Discussão}

Utilizando a base de artigos Web of Science e Scopus, empregou-se como palavra-chave o gênero Malpighia, e a espécie Malpighia emarginata, resultando em 169 e 179 artigos indexados, respectivamente (Tabela 1). Refinando a pesquisa para a espécie (Malpighia emarginata) direcionada para áreas tecnológicas, obteve-se um total de 47 e 36 para "food", 17 e 17 para "genetic", 21 e 20 para "production" e 37 e 33 para "acid ascórbic" para a SCOPUS e Web of Science, respectivamente. Os bancos de patentes registram um número menor de documentos em que nas bases EPO e WIPO foram depositadas 11 e 20 patentes para Malpighia emarginata, respectivamente; e "Malpighia emarginata AND food" foram contabilizados dois documentos 
para ambas a bases WIPO e EPO, respectivamente. As tecnologias produzidas com os termos "production" e "acid ascorbic" foram um documento para cada. No banco de dados do Instituto Nacional da Propriedade Industrial (INPI) foram registrados apenas dois documentos para a espécie.

Tabela 1 - Número de artigos e patentes registrados por base de dados envolvendo os termos utilizados

\begin{tabular}{cccccc} 
DEScRITORES & WEB OF SCIENCE & SCOPUS & EPO & WIPO & INPI \\
Malpighiaceae & 504 & 727 & 5 & 6 & 2 \\
\hline Malpighia emarginata & 169 & 179 & 11 & 20 & 2 \\
\hline Malpighia emarginata AND food & 36 & 47 & 2 & 2 & - \\
\hline Malpighia emarginata AND genetic & 17 & 17 & - & - & - \\
\hline Malpighia emarginata AND production & 20 & 21 & 1 & - & - \\
\hline Malpighia emarginata AND ascorbic acid & 33 & 37 & - & 1 & -
\end{tabular}

Fonte: Elaborada pelos autores deste artigo (2019)

Considerando que as bases utilizadas possuíram número significativo de documentos sobre a espécie e as diversas áreas de aplicações tecnológicas, a pesquisa foi norteada no sentido de explorar melhor as informações que essas bases pudessem fornecer em relação à distribuição de documentos por país, ano, área de publicação de artigos e Classificação Internacional de Patentes (CIP). E, além dos dados quantitativos, foi possível prospectar sobre as áreas mais importantes aplicadas da espécie a partir das pesquisas já realizadas.

\section{Prospecção Tecnológica}

Com relação a países, a China $(\mathrm{EPO}=5$ e WIPO=6) e a Coreia do Sul $(\mathrm{EPO}=3$ e WIPO=4) são os maiores detentores de patentes envolvendo o desenvolvimento de pesquisas sobre Malpighia emarginata (Figura 1), seguidos do Brasil, Estados Unidos, Japão e Austrália. Na base EPO e WIPO, a maioria das patentes está depositada em países de origem asiática como China, República da Coreia e Japão. Apesar da espécie ter pouca ocorrência e produção nesses países, os estudos sobre ela têm avançado e, assim, a população mundial tem descoberto as potencialidades e a importância da planta por meio de publicações e de patentes oficializadas nas bases internacionais (NOGUEIRA et al., 2019). Esse fato é de ampla relevância para o conhecimento da espécie e para novas alternativas saudáveis em diversos setores, constatando uma fonte de renda potencial (MARTINS et al., 2016). 
Figura 1 - Distribuição por país de patentes depositadas na EPO e WIPO utilizando o termo Malpighia emarginata

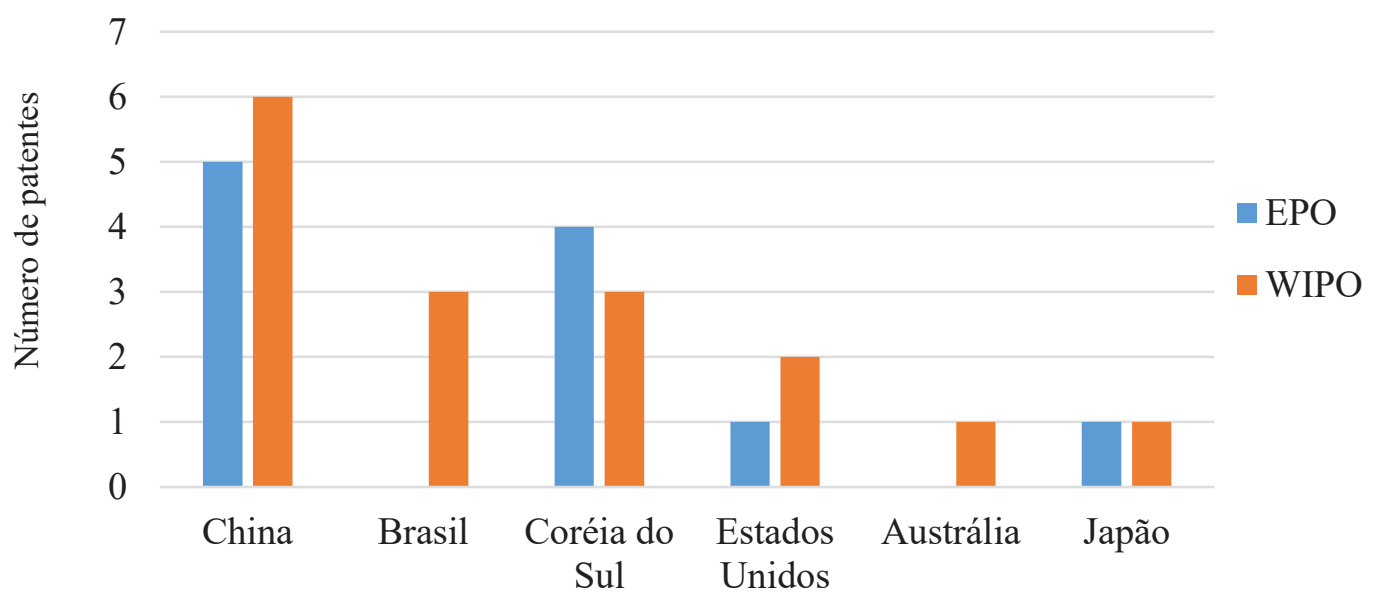

Fonte: Elaborada pelos autores deste artigo (2019)

A partir da busca com o a palavra-chave "Malpighia emarginata" na EPO e no WIPO, observou-se que os depósitos se iniciaram em 2004 (Figura 2) com um grande número de tecnologias registradas a partir de 2016, o que aponta 59,37\% das patentes depositadas de 2016 até o presente momento. No ano de 2018, foi registrado o maior número de patentes envolvendo a aceroleira nas bases EPO (4) e WIPO (7).

Figura 2 - Evolução anual de depósitos de patentes na EPO e WIPO utilizando o termo Malpighia emarginata

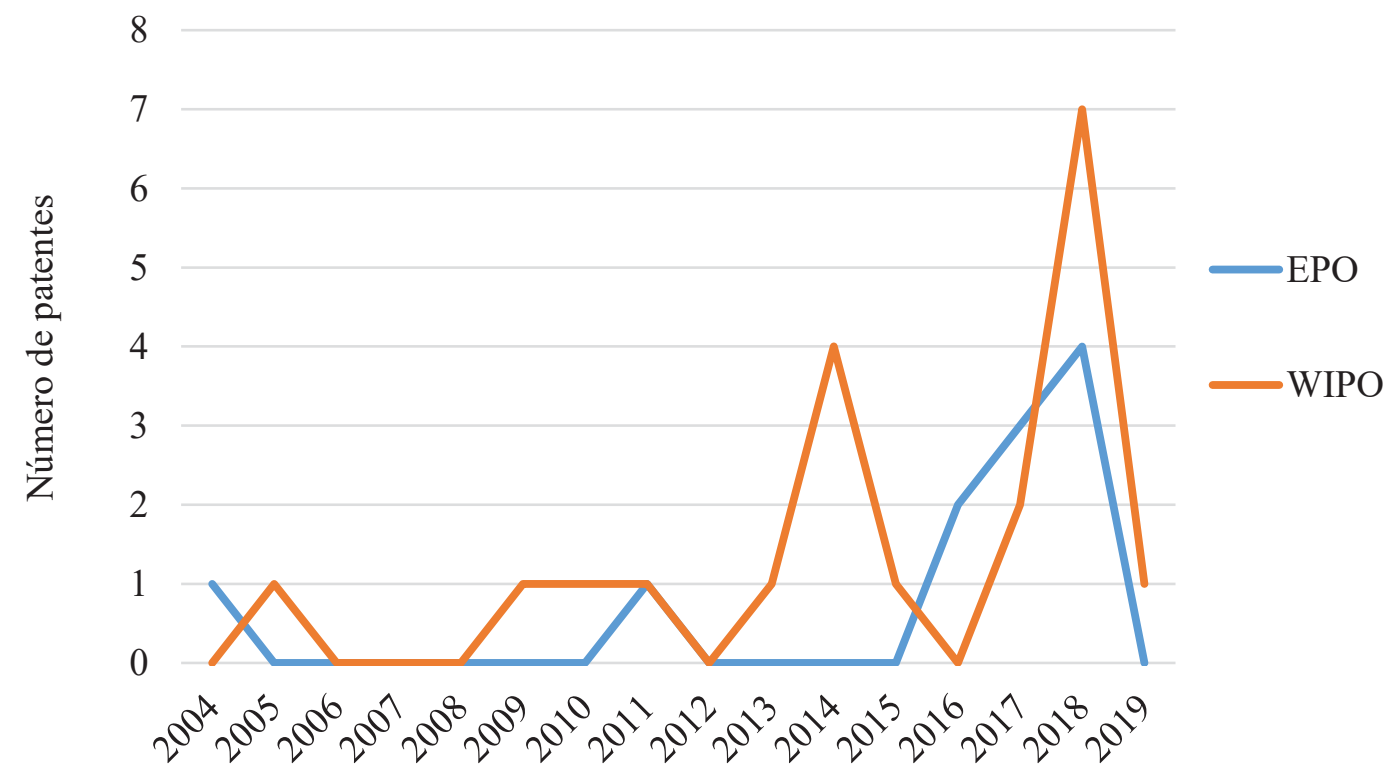

Fonte: Elaborada pelos autores deste artigo (2019) 
Em se tratando de uma prospecção tecnológica, um formato importante para acelerar buscas nas bases patentearias é a Classificação Internacional de Patentes (CIP), na qual as patentes são classificadas de acordo com a aplicação. São divididas em oito seções, 21 subseções, 120 classes, 628 subclasses e 69.000 grupos (SERAFINI et al., 2012).

Analisando a CIP das patentes depositadas nas bases WIPO e EPO, verificou-se que todas correspondem unicamente à Seção A (Figura 3), que corresponde às necessidades humanas. As Subclasses mais presentes são A23L ( $\mathrm{EPO}=7$ e $\mathrm{WIPO}=8)$ e $\mathrm{A61K}(\mathrm{EPO}=5$ e WIPO=16). A Subclasse A23L compreende os produtos alimentícios e as bebidas (cinco patentes), como a invenção de um produto que contém carne in natura com o fruto de acerola, sendo alternativa para alimentação nutricional e mais equilibrada, segundo o inventor Russell (RUSSEL; NUNEZ, 2018). Já a subclasse A61K está voltada para finalidades médicas, odontológicas ou higiênicas, contém quatro patentes com essa classificação.

Figura 3 - Distribuição por CIP dos depósitos de patentes na EPO e WIPO utilizando o termo Malpighia emarginata

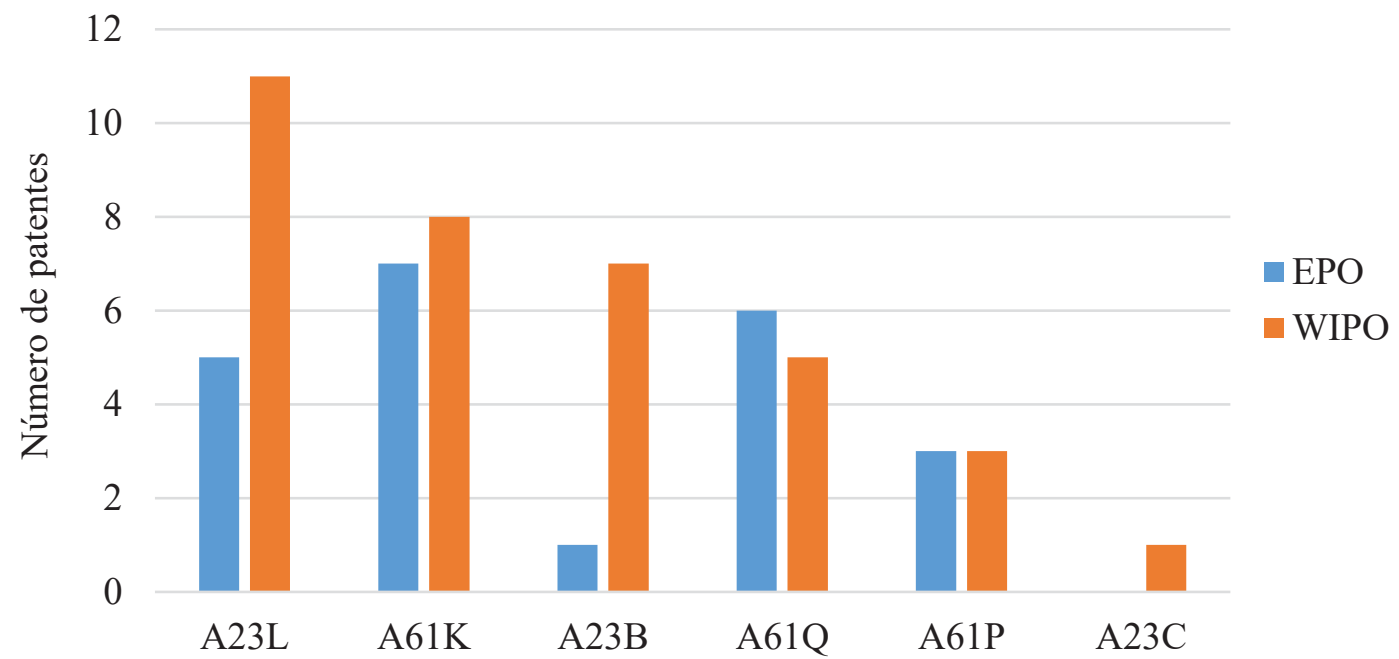

Fonte: Elaborada pelos autores deste artigo (2019)

\section{Prospecção Científica}

O Brasil é o país com o maior número de pesquisas científicas que resultaram em publicações indexadas em ambas as bases utilizadas, com larga vantagem, apresentando 154 e 138 publicações nas bases SCOPUS e Web of Science (Figura 4), o que representa mais de $80 \%$ em ambas as bases. Japão, Espanha, Estados Unidos, Alemanha e Índia apresentaram em menor número publicações com aceroleira. Esse resultado se deve ao fato de se tratar de uma planta tropical, que apresenta fruto de grande potencial econômico, nutricional, medicinal e ecológico, e que se destaca em relação às outras espécies de igual potencial, principalmente pela sua adaptabilidade ambiental (FREITAS et al., 2014). A acerola é um fruto climatérico, com elevado pico da taxa respiratória (900 $\mathrm{mL} \mathrm{CO}_{2} \mathrm{~kg}-1 \mathrm{~h}$ ), o que explica muito quanto ao destaque que o Brasil oferece (CARRINGTON; KING, 2002; DE MORAES et al., 2017).

Apesar de exótica, a aceroleira consegue adaptar-se bem ao Brasil por ser um país de clima tropical, proporcionando uma boa produção de frutos, com colheita que pode ser realizada 
em quase todas as épocas do ano (FURLANETO; NASSER, 2015), o que justifica o mercado interno estar investindo cada vez mais nas potencialidades dessa frutífera, proporcionando uma alternativa de renda para muitos agricultores do país (DE JESUS, 2003; BEZERRA et al. , 2017).

Figura 4 - Distribuição por país de artigos indexados na base SCOPUS e Web of Science com Malpighia emarginata

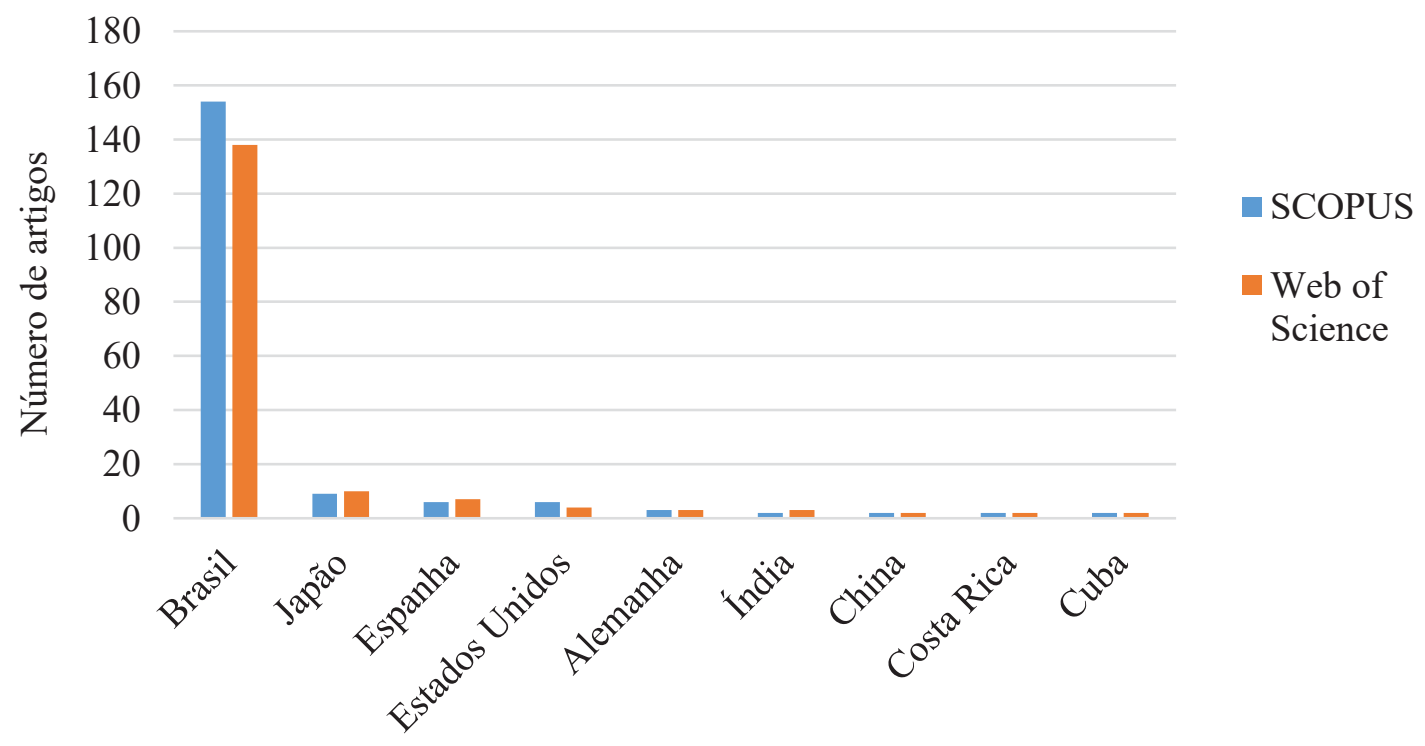

Fonte: Elaborada pelos autores deste artigo (2019)

A Figura 5 representa a evolução das publicações indexadas na base de artigos Scopus e Web of Science com a espécie Malpighia emarginata. As publicações começaram em 1999 na base Scopus e Web of Science, respectivamente, com maior número de trabalhos reportado para o ano de 2018 ( $\mathrm{n}=22$ ). Houve um aumento do número de publicações nesta última década em relação à primeira década dos anos 2000 , com 75,73\% e 66,32\% dos documentos indexados na última década nas bases SCOPUS e Web of Science, respectivamente.

Quanto ao número de publicações sobre a espécie (Figura 5), é possível verificar que, em 2018 , houve maior produção científica $(n=22)$, isso comparado aos outros anos. Em 2018 , os artigos foram impulsionados pelo crescimento de pesquisas que envolviam a atividade antioxidante do grupo (BATISTA et al., 2018; KLOSTERHOFF et al., 2018; CARMO; NAZARENO; RUFINO, 2018). Os estudos demostraram grande valor antioxidante do fruto da aceroleira $e$ o alto valor funcional presente em fibras, tudo isso aliado à atividade antioxidante demonstra um grande valor agregado na promoção de produtos com propriedades benéficas para a saúde (CARMO; NAZARENO; RUFINO, 2018). Por outro lado, a preocupação em garantir a segurança alimentar e em prolongar a vida útil de vitaminas presentes na aceroleira, principalmente a vitamina $\mathrm{C}$, tem levado a alternativas como a utilização do processamento ultrassônico para descontaminar o suco de frutas (SANTOS; RODRIGUES; FERNANDES, 2018). Os históricos de publicações também abordam temas como polinização, propagação da espécie, produção de mudas, nutrientes e minerais encontrados na planta. A busca em massa por pesquisas sobre a produção de acerola vem sendo cada vez mais atualizada, pois a produção dessa planta é muito importante para o nosso país, já que aqui somos os maiores consumidores e exportadores dessa fruta (UCHÔA et al., 2017). 
Figura 5 - Evolução anual de artigos indexados na base SCOPUS e Web of Science com Malpighia emarginata

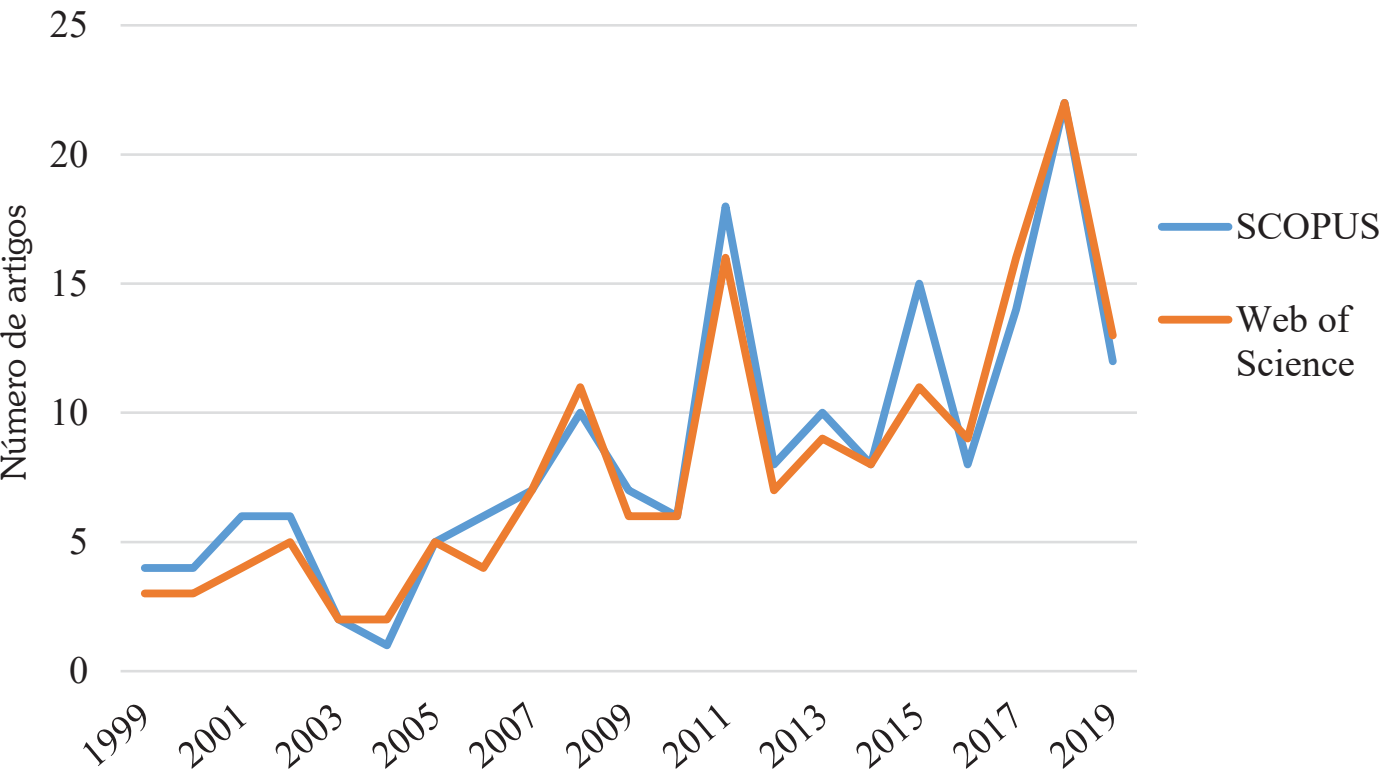

Fonte: Elaborada pelos autores deste artigo (2019)

Ao verificar as principais áreas de publicação de artigos na SCOPUS e Web of Science sobre Malpighia emarginata, constata-se que a área de Agricultura e Ciências Biológicas surge em primeiro lugar com 54,17\% (Tabela 2), seguida da Bioquímica, Genética e Biologia Molecular e Química, com 12,88\% e 10,98\%, respectivamente. A concentração de publicações em áreas voltadas para a produção visando ao mercado alimentício corrobora com a evolução e a valorização da aceroleira na área alimentícia, colocando o Brasil como o principal exportador do fruto (FREITAS et al., 2014).

As áreas com menor resultado são Farmacologia e Veterinária (Tabela 2). Pesquisas na área de cosmetologia indicam a inclusão de ácido ascórbico em produtos contra o envelhecimento celular devido à ação antioxidante da espécie, pela retenção de radicais livres, e pelas proteínas serem responsáveis pelas ações de hidratação e condicionamento capilar (NEGRI, 2016). No mercado, entretanto, é mais valorizada na área alimentícia. Poucas pesquisas valorizando o potencial farmacológico justificam o resultado obtido para área Farmacológica. As pesquisas voltadas para essa área abordam temas como extração do fruto e aplicação da ação oxidante em medicamentos e cosméticos (NOGUEIRA et al., 2018).

O Brasil, além de ser o terceiro maior produtor mundial de frutas, em 2017, registrou a produção de 43,5 milhões de toneladas, sendo que $10 \%$ desse valor contribuiu para a produção mundial (KIST et al., 2018). Malpighia emarginata se destaca cada vez mais, pois o país é o que mais produz e a exporta, com total aproximado de 33.000 toneladas por ano, cultivadas em aproximadamente 11.000 hectares (DE OLIVEIRA; NICODEMO; DE OLIVEIRA, 2015). Vale ressaltar que a aceroleira é uma planta que consegue se adaptar às mais diferentes condições climáticas e, quando bem manejada, consegue florescer e frutificar várias vezes no ano (FURLANETO; NASSER, 2015), o que explica a quantidade de artigos na área da Agricultura (Tabela 2). 
Tabela 2 - Distribuição com das publicações indexadas por áreas na base SCOPUS e Web Of Science com Malpighia emarginata

\begin{tabular}{ccc} 
ÁreAs DE INTERESSE & SCOPUS E & WEB OF ScIENCE \\
Agricultura e ciências biológicas & 143 & 50,88 \\
\hline Bioquímica, genética e biologia molecular & 34 & 12,09 \\
\hline Química & 29 & 10,32 \\
\hline Engenharia & 12 & 4,27 \\
\hline Engenharia química & 11 & 3,91 \\
\hline Medicina & 10 & 3,55 \\
\hline Ciências ambientais & 9 & 3,20 \\
\hline Imunologia e microbiologia & 6 & 2,13 \\
\hline Farmacologia, toxicologia e farmacêutica & 5 & 1,77 \\
\hline Veterinária & 5 & 1,77 \\
\hline
\end{tabular}

Fonte: Elaborada pelos autores deste artigo (2019)

\section{Considerações Finais}

As pesquisas tecnológicas e científicas sugerem que o gênero Malpighia tornou-se bastante conhecido devido às suas propriedades nutricionais. A China é o país com o maior número de patentes depositadas sobre aceroleira, no entanto, o Brasil apresenta-se com o maior número de artigos científicos. As patentes (CIP) e os artigos científicos foram incluídos na área alimentícia, o que coloca o Brasil com amplo potencial nesse setor, visto que o país é atualmente o maior exportador de produtos a partir de acerola do mundo. As pesquisas científicas tiveram início por volta dos anos 2000, com grande evolução nas últimas duas décadas, fator impulsionado pela aplicação de pesquisas com o táxon em outras áreas como: farmacologia, genética e química. Apesar de o Brasil produzir um grande número de pesquisas com o grupo, é necessário que haja maior produção de tecnologias, fator preponderante para avanços técnicos científicos com aplicação de Malpighia emarginata, espécie importante economicamente para o Brasil.

\section{Referências}

ACIOLI, G. R.; ABUD, A. K. S.; JUNIOR, A. M. O. Prospecção Tecnológica da Indústria Farmacêutica no Brasil. In: ENCONTRO NORDESTINO DE ETNOBIOLOGIA E ETNOECOLOGIA, 1995, Aracajú, Anais [...]. Aracajú: Embrapa Tabuleiros Costeiros, 2015. v. 3, n.1, p. 185-191.

AGÊNCIA PAULISTA DE TECNOLOGIA DOS AGRONEGÓCIOS. Agregação de valor e articulação da cadeia de produção consolidam o mercado de acerola na Alta Paulista. São Paulo, [2007]. Disponível em: http://www.apta.sp.gov.br/noticias. Acesso em: 24 dez. 2019.

ANDERSON, W. R. Origins of Mexican Malpighiaceae. Acta Botanica Mexicana, [S.l.], v. 104, n. 1, p. 107-156, 2013. 
ARAÚJO, S. S. et al. Prospecção de patentes biotecnológicas com óleo essencial em microemulsão como agente anti-inflamatório. Revista GEINTEC, [S.l.], v. 5, n. 2, p. 2.058-2.065, 2015.

BASF, S. A. Unidades de Produtos para Fruticultura. Frutas para exportação. Atualidades agrícolas: fruticultura o sucesso do Vale São Francisco, São Bernardo do Campo, n. 6, p. 1629, jun., 2006.

BATISTA, Kamila Sabino et al. Beneficial effects of consumption of acerola, cashew or guava processing by-products on intestinal health and lipid metabolism in dyslipidaemic female Wistar rats. British Journal of Nutrition, [S.I.], v. 119, n. 1, p. 30-41, 2018.

BELWAL, T. et al. Phytopharmacology of Acerola (Malpighia spp.) and its potential as functional food. Trends Food Sci. Technol., [S.l.], p. 74, 99-106, 2018.

BEZERRA, A. S. et al. Produção de mudas de acerola (Malpighia emarginata DC.) pelo método de enxertia em topo por garfagem em fenda cheia. Revista Agroecossistemas, [S.l.], v. 9, n. 1, p. 251-260, 2017.

CARMO, J. S.; NAZARENO, L. S. Q.; RUFINO, M. S. M. Characterization of the acerola industrial residues and prospection of their potential application as antioxidant dietary fiber source. Food Science and Technology, [S.l.], v. 38, p. 236-241, 2018.

CARRINGTON, C. M. S.; KING, R. A. G. Fruit development and ripenig in Barbados cherry, Malpighia emarginata D.C. Scientia Horticulturae, [S.l.], v. 92, n. 1, p. 1-7, 2002.

CAVALCANTI, D. T. B. Desenvolvimento de fruta estruturada simples e mista com ciriguela e acerola. 2012. 168 p. Dissertação (Ciência e Tecnologia de Alimentos) - Universidade Federal Rural de Pernambuco, Recife, 2012.

DA CRUZ, E. S. et al. Diversidade genética de aceroleiras (Malpighia emarginata) com base em descritores morfoagronômicos. In: CONGRESSO BRASILEIRO DE MELHORAMENTO DE PLANTAS, 6., 2011. Embrapa Mandioca e Fruticultura - Artigo em anais de congresso (ALICE). 2011.

DE ALMEIDA, G. F. et al. Floração e frutificação da aceroleira (Malphigia emarginata DC.) em uma área no semiárido Brasileiro. Magistra, [S.l.], v. 26, n. 2, p. 242-248, 2017.

DE JESUS, M. F. et al. Produção de passas de acerola em secador de bandeja. Revista Brasileira de Produtos Agroindustriais, [S.l.], v. 5, n. 1, p. 81-87, 2003.

DE MORAES, B. S. R. et al. Resposta ao etileno e alterações pós-colheita de acerolas flor branca. In: JORNADA DE INTEGRAÇÃO DA PÓS-GRADUAÇÃO DA EMBRAPA SEMIÁRIDO, 2., 2017. Embrapa Semiárido - Artigo em anais de congresso (ALICE). 2017.

DE OLIVEIRA, J. E. M.; NICODEMO, D.; DE OLIVEIRA, F. F. Contribuição da polinização entomófila para a produção de frutos de aceroleira. Pesquisa Agropecuária Tropical, [S.l.], v. 45, n. 1, p. 56-65, 2015.

DELVA, L.; GOODRICH $\square$ SCHNEIDER, R. Antioxidant activity and antimicrobial properties of phenolic extracts from acerola (Malpighia emarginata $D C$ ) fruit. International Journal of Food Science \& Technology, [S.l.], v. 48, n. 5, p. 1.048-1.056, 2013.

DOS SANTOS, P. T. V. et al. Detecção de Meloidogyne enterolobii em mudas de amoreira (Morus nigra L.). Ciência Rural, [S.l.], v. 45, n. 5, p. 757-759, 2015. 
EPO, K. M.; KAMEYAMA, H. Antioxidant and skin care preparation for external use, using the same and method for activating cell by using the same. 2005220085, 18 ago. 2005.

EPO. XICHEN, P. Blueberry Lutein. 106418546, 22 dez. 2017.

FAO, 2010. Produtividade das culturas do mundo. Disponível em: http://faostat.fao.org/. Acesso em: 14 dez. 2019.

FREITAS, C. A. S. et al. Acerola: produção, composição, aspectos nutricionais e produtos. Current Agricultural Science and Technology, [S.1.], v. 12, n. 4, 2014.

FURLANETO, F. P. B.; NASSER, M. D. Panorama da cultura da acerola no estado de São Paulo. Pesquisa \& Tecnologia, [S.l.], v. 12, n. 1, 2015.

GUIA IPC. Guia de Classificação Internacional de Patentes. 2019. Disponível em: http://ipc. inpi.gov.br/. Acesso em: $1^{\circ}$ jul. 2019.

JESUS, A. L. N. de; LEMOS, A. C. G. Elaboração e avaliação sensorial de barra de cereal enriquecida com farinha de acerola (Malpighia glabra L.). 2018. 31p. Trabalho de Conclusão de Curso (Nutrição) - Centro Universitário Toledo, Araçatuba, SP, 2018.

JUNIOR, R. G. O. et al. Prospecção tecnológica do gênero Annona (Annonaceae). Revista GEINTEC - Gestão, Inovação e Tecnologias, [S.I.], v. 4, n. 2, p. 850-858, 2014.

KIST, Benno Bernardo et al. Anuário brasileiro da fruticultura 2018. Santa Cruz do Sul: Editora Gazeta Santa Cruz, v. 88, p. 2.018, 2018.

KLOSTERHOFF, R. R. et al. Structure and intracellular antioxidant activity of pectic polysaccharide from acerola (Malpighia emarginata). International Journal of Biological Macromolecules, [S.l.], v. 106, p. 473-480, 2018.

MALPIGHIACEAE in Flora do Brasil 2020 em construção. Jardim Botânico do Rio de Janeiro. [2019]. Disponível em: http://floradobrasil.jbrj.gov.br/reflora/floradobrasil/FB155. Acesso em: $1^{\circ}$ jul. 2019.

MARQUES, L G. A. et al. Desenvolvimento do potencial tecnológico de Casearia sylvestris. Revista GEINTEC - Gestão, Inovação e Tecnologias, [S.I.], v. 3, n. 4, p. 70-85, 2013.

MARTINS, E. A. et al. Rentabilidade da produção de acerola orgânica sob condição determinística e de risco: estudo do distrito de irrigação Tabuleiro Litorâneo do Piauí. Revista de Economia e Sociologia Rural, [S.I.], v. 54, n. 1, p. 9-28, 2016.

MENEZES, A. R. V. et al. Estudo Comparativo do Pó da Acerola Verde (Malphigia emarginata DC.) Obtido em Estufa por Circulação de Ar e por Liofilização. Revista Brasileira de Produtos Agroindustriais, [S.l.], v. 11, n. 1, p. 1-8, 2009.

NEGRI, T. C.; BERNI, P.; BRAZACA, S. Valor nutricional de frutas nativas e exóticas do Brasil. Biosaúde, [S.1.], v. 18, n. 2, p. 82-96, 2016.

NETO, A. F. et al. Determinação de vitamina ce avaliação físico-química em três variedades de acerola cultivadas em Petrolina-PE. Nucleus, [S.l.], v. 11, n. 1, 2014.

NOGUEIRA, G. D. R. et al. Analysis of a hybrid packed bed dryer assisted by infrared radiation for processing acerola (Malpighia emarginata DC.) residue. Food and Bioproducts Processing, [S.l.], v. 114, p. 235-244, 2019. 
NOGUEIRA, R. F. et al. Vitamina C: uso tópico no tratamento do melasma e envelhecimento precoce. Mostra Científica da Farmácia, [S.l.], v. 4, n. 2, 2018.

OLIVEIRA, M. G. et al. Diversidade genética de aceroleiras (Malphigia emarginata DC), utilizando marcadores moleculares RAPD e características morfoagronômicas. Revista Brasileira de Fruticultura, [S.l.], v. 31, n. 1, p. 162-170, 2009.

QUINTELLA, C. M. et al. Prospecção tecnológica como uma ferramenta aplicada em ciência e tecnologia para se chegar à inovação. Revista Virtual de Química, [S.l.], v. 3, n. 5, p. 406-415, 2011.

REZENDE, Y. R. R. S.; NOGUEIRA, J. P.; NARAIN, N. Comparison and optimization of conventional and ultrasound assisted extraction for bioactive compounds and antioxidant activity from agroindustrial acerola (Malpighia emarginata DC) residue. LWT - Food Science and Technology, [S.I.], 85, p. 158-169, 2017.

RITZINGER, R.; RITZINGER, C. H. S. P. Acerola. Embrapa Mandioca e Fruticultura-Artigo em periódico indexado (ALICE), 2011.

RUSSELL, Lanzrath. NUNEZ, Maisonet Wigberto. Produtos à base de carne. CN103997916. Patente. 2018.

SANTOS, J. C. R. et al. Ensaios preliminares para produção de estruturados com Acerola e Ciriguela. Revista do Congresso Sul Brasileiro de Engenharia de Alimentos, [S.l.], 2016.

SANTOS, Valéria; RODRIGUES, Sueli; FERNANDES, Fabiano. Improvements on the stability and vitamin content of acerola juice obtained by ultrasonic processing. Foods, [S.l.], v. 7, n. 5, p. 68, 2018.

SERAFINI, M. R. et al. Mapeamento de tecnologias patenteáveis com o uso da hecogenina. Revista Geintec, [S.I.], v. 2, n. 5, p. 427-435, 2012.

SHINOHARA, N. K. S. et al. A Mãe da Acerola (Malpighia glabra L.) no Brasil. Revista Eletrônica “Diálogos Acadêmicos", [S.I.], v. 9, n. 2, p. 49- 63, 2015.

SILVA, N. S. Avaliação Econômica de Sistemas de Irrigação Localizada na Acerola. In: IV WINOTEC - WORKSHOP INTERNACIONAL DE INOVAÇÕES TECNOLÓGICAS NA IRRIGAÇÃO, 2012.

Anais [...], 2012.

SILVA, P. B. et al. Uso de secador roto-aerado com pré-tratamento na desidratação de resíduos de acerola. Blucher Chemical Engineering Proceedings, [S.I.], v. 1, n. 2, p. 5.537-5.544, 2015.

SILVA, P. C. C. et al. Crescimento de mudas de Malpighia emarginata em diferentes substratos. Engenharia Ambiental: Pesquisa e Tecnologia, [S.l.], v. 13, n. 2, 2016.

SPÓSITO, R. C. A.; VIRGENS, R. S.; PUNGARTNIK, Cristina. Investigação prospectiva sobre a utilização do gênero Cyperus na geração de tecnológicas. Cadernos de Prospecção, [S.l.], v. 9, n. 1, p. 38, 2016.

TACO. Tabela brasileira de composição de alimentos. 4. ed. Campinas: UNICAMP, 2011. 36p.

UCHÔA, V. T. et al. avaliação do teor de vitamina c em polpas de acerola comercializadas em supermercados de Piripiri-PI. Revista Ciência Agrícola, [S.I.], v. 15, n. 1, p. 59-68, 2017.

WIPO - INTERNATIONAL PATENT CLASSIFICATION (Version 2016.1). WIPO. Disponível em: http://www.wipo.int/portal/en/index.html. Acesso em: 3 dez. 2019. 


\section{Sobre os Autores}

\section{Thaynara de Carvalho Vieira}

E-mail: thataasd@outlook.com

Graduanda em Ciências Biológicas pela Universidade Federal do Delta do Parnaíba (UFDPar).

Endereço profissional: Universidade Federal do Delta do Parnaíba (UFDPar), Av. São Sebastião, n. 2.819, Nossa

Sra. de Fátima, Parnaíba, PI. CEP: 64202-020.

\section{Maria Gracelia Paiva Nascimento}

E-mail: graceliapaiva@gmail.com

Doutoranda em Desenvolvimento e Meio Ambiente (PRODEMA/UFPI/TROPEN) pela Universidade Federal do Piauí (UFPI).

Endereço profissional: Av. Universitária, n. 1.310, Bairro Ininga, Teresina, PI. CEP: 64049-550.

\section{Cleiton Barroso Bittencourt}

E-mail: cleiton_court@hotmail.com

Doutorando em Biotecnologia Vegetal pela Universidade Federal de Lavras (UFLA).

Endereço profissional: Universidade Federal de Lavras (UFLA), Campus Universitário, s/n, Lavras, MG. CEP: 37200-000.

\section{Ivanilza Moreira de Andrade}

E-mail: ivanilzaandrade@hotmail.com

Doutora em Botânica pela Universidade Estadual de Feira de Santana (UEFS) (2006). Atualmente é docente da Universidade Federal do Delta do Parnaíba (UFDPar).

Endereço profissional: Universidade Federal do Delta do Parnaíba (UFDPar), Av. São Sebastião, n. 2.819, Nossa Sra. de Fátima, Parnaíba, PI. CEP: 64202-020. 